\title{
POSSIBLE SIGNS OF WATER AND DIFFERENTIATION IN A ROCKY EXOPLANETARY BODY
}

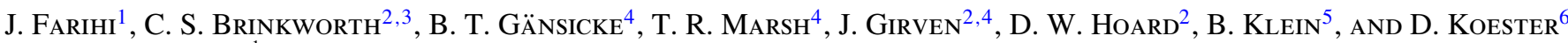 \\ ${ }^{1}$ Department of Physics \& Astronomy, University of Leicester, Leicester LE1 7RH, UK; jf123@ star.le.ac.uk \\ ${ }^{2}$ Spitzer Science Center, California Institute of Technology, MS 220-6, Pasadena, CA 91125, USA \\ ${ }^{3}$ NASA Exoplanet Science Institute, California Institute of Technology, MS 100-22, Pasadena, CA 91125, USA \\ ${ }^{4}$ Department of Physics, University of Warwick, Coventry CV5 7L, UK \\ ${ }^{5}$ Department of Physics \& Astronomy, University of California, Los Angeles, CA 90095, USA \\ ${ }^{6}$ Institut für Theoretische Physik und Astrophysik, University of Kiel, 24098 Kiel, Germany \\ Received 2010 December 8; accepted 2010 December 30; published 2011 January 18
}

\begin{abstract}
Spitzer observations reveal the presence of warm debris from a tidally destroyed rocky and possibly icy planetary body orbiting the white dwarf GD 61. Ultraviolet and optical spectroscopy of the metal-contaminated stellar photosphere reveal traces of hydrogen, oxygen, magnesium, silicon, iron, and calcium. The nominal ratios of these elements indicate an excess of oxygen relative to that expected from rock-forming metal oxides, and thus it is possible that water was accreted together with the terrestrial-like debris. Iron is found to be deficient relative to magnesium and silicon, suggesting the material may have originated as the outer layers of a differentiated parent body, as is widely accepted for the Moon.
\end{abstract}

Key words: circumstellar matter - minor planets, asteroids: general - planetary systems - stars: abundances white dwarfs

Online-only material: color figures

\section{INTRODUCTION}

In the search for terrestrial planetary systems around other stars, white dwarfs offer a unique astrophysical advantage. Owing to high surface gravities and the onset of convection, any atmospheric metals sink rapidly as these Earth-sized stellar embers cool below 25,000 K, leaving behind only $\mathrm{H}$ or $\mathrm{He}$ in the outermost layers of the star (Koester 2009), a physical process corroborated by observation (Koester et al. 2005; Zuckerman et al. 2003). Those stars with rocky planetary system remnants can become contaminated by the accretion of small, but spectroscopically detectable, amounts of heavy elements. Metal lines in cool white dwarfs are a telltale of external pollution that typically implies either ongoing metal accretion rates $\dot{M}_{\mathrm{z}}>10^{8} \mathrm{~g} \mathrm{~s}^{-1}$ (Koester \& Wilken 2006) or asteroid-sized masses of heavy elements within the convection zone of the star (Farihi et al. 2010a).

To date, metal-rich dust and gas disks (Jura et al. 2009a; Gänsicke et al. 2008, 2006; Reach et al. 2005), very likely produced by the tidal disruption of large asteroids (Debes \& Sigurdsson 2002), have been found closely orbiting more than one dozen cool white dwarfs (Farihi et al. 2009; von Hippel et al. 2007; Jura et al. 2007) and provide a ready explanation for the metal absorption features seen in their atmospheres (Jura 2003). The circumstellar material being gradually accreted by the white dwarf can be directly observed in the stellar photosphere to reveal its elemental abundances (Klein et al. 2010; Zuckerman et al. 2007). While transit spectroscopy of main-sequence stars offers some information on the chemical composition of extrasolar gas giant atmospheres, metal-polluted white dwarfs distill entire rocky planetary bodies into their constituent elements.

This Letter reports the detection of circumstellar dust at the white dwarf GD 61, an He-rich star whose atmosphere is highly polluted with $\mathrm{O}, \mathrm{Si}$, and $\mathrm{Mg}$ and to a lesser degree by $\mathrm{Fe}$ and $\mathrm{Ca}$. With the exception of $\mathrm{Mg}$ detected in this work, these photospheric heavy elements were identified previously via farultraviolet (Desharnais et al. 2008) and optical (Sion et al. 1988) spectroscopy, but interpreted as products of convective dredge-up and interstellar accretion. The analysis here reinterprets these elements as the result of infall from closely orbiting circumstellar debris that was once contained in a sizable planetary body such as a large asteroid.

\section{DATA AND ANALYSIS}

\subsection{Circumstellar Dust}

Spitzer Space Telescope (Werner et al. 2004) observations of GD 61 were executed on 2009 March 18 and April 7 during the telescope's final cryogenic cycle. Imaging photometry was performed with the Infrared Array Camera (IRAC; Fazio et al. 2004) at 4.5 and $7.9 \mu \mathrm{m}$, and with the Infrared Spectrograph (IRS; Houck et al. 2004) in the blue peak-up filter. The data were reduced and photometry executed following Farihi et al. (2009) and Brinkworth et al. (2009); results are listed in Table 1 and plotted in Figure 1.

The flux densities measured with Spitzer exhibit an infrared excess, consistent with warm dust orbiting within the tidal breakup (Roche) limit of the star (von Hippel et al. 2007). These data cannot be reproduced with a low-mass stellar or brown dwarf secondary (Patten et al. 2006; Farihi et al. 2005). In Figure 1, a model stellar atmosphere is fitted to the groundbased photometry, and the addition of an optically thick disk model with a flat geometry is shown to reproduce the IRAC data rather well. The measured $15.8 \mu \mathrm{m}$ IRS peak-up flux is likely to contain silicate emission, as observed in G29-38 (Reach et al. 2009), and strongly suspected in SDSS 1228 (Brinkworth et al. 2009).

The temperatures of the disk model correspond to inner and outer disk edges of 19 and 26 stellar radii $(0.21$ and $\left.0.30 R_{\odot}\right)$, respectively. Thus, the dust ring is relatively narrow $\left(\Delta r<0.1 R_{\odot}<r_{\text {inner }}\right)$ as found for a growing number of 


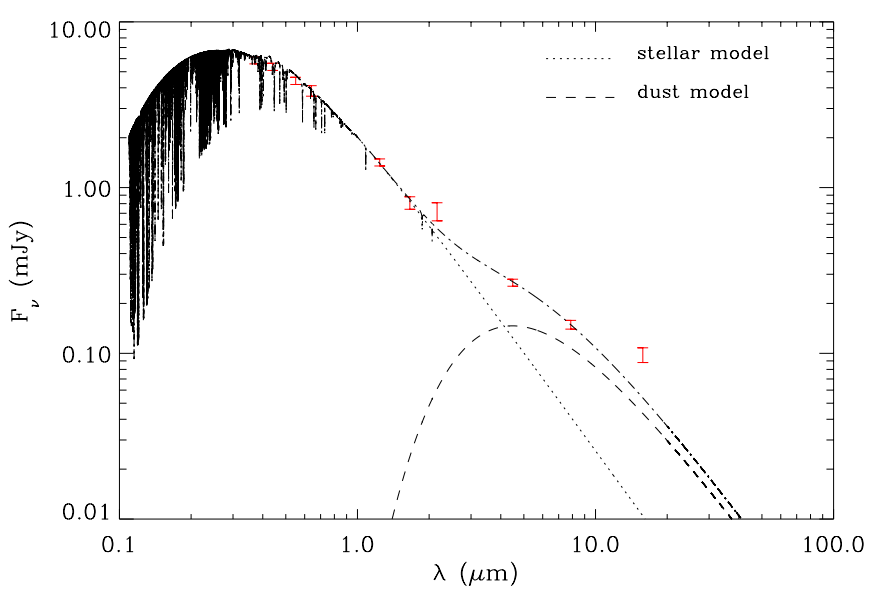

Figure 1. Ultraviolet through infrared energy distribution of GD 61 with photometry represented as error bars. Fluxes below $2 \mu \mathrm{m}$ (the 2MASS $K_{s}$-band measure appears inaccurate and has been ignored) are fitted with a model atmosphere, yielding a photometric distance of $50.1 \mathrm{pc}$. The dashed line is a flat disk model with inner and outer temperatures of 1300 and $1000 \mathrm{~K}$ at $i=$ $79^{\circ}$. The IRS blue peak-up flux is likely to contain significant silicate emission. (A color version of this figure is available in the online journal.)

metal-polluted white dwarfs (Farihi et al. 2010b). Otherwise, the infrared continuum at GD 61 is fairly typical of white dwarf disks studied with Spitzer (Farihi et al. 2009; Jura et al.
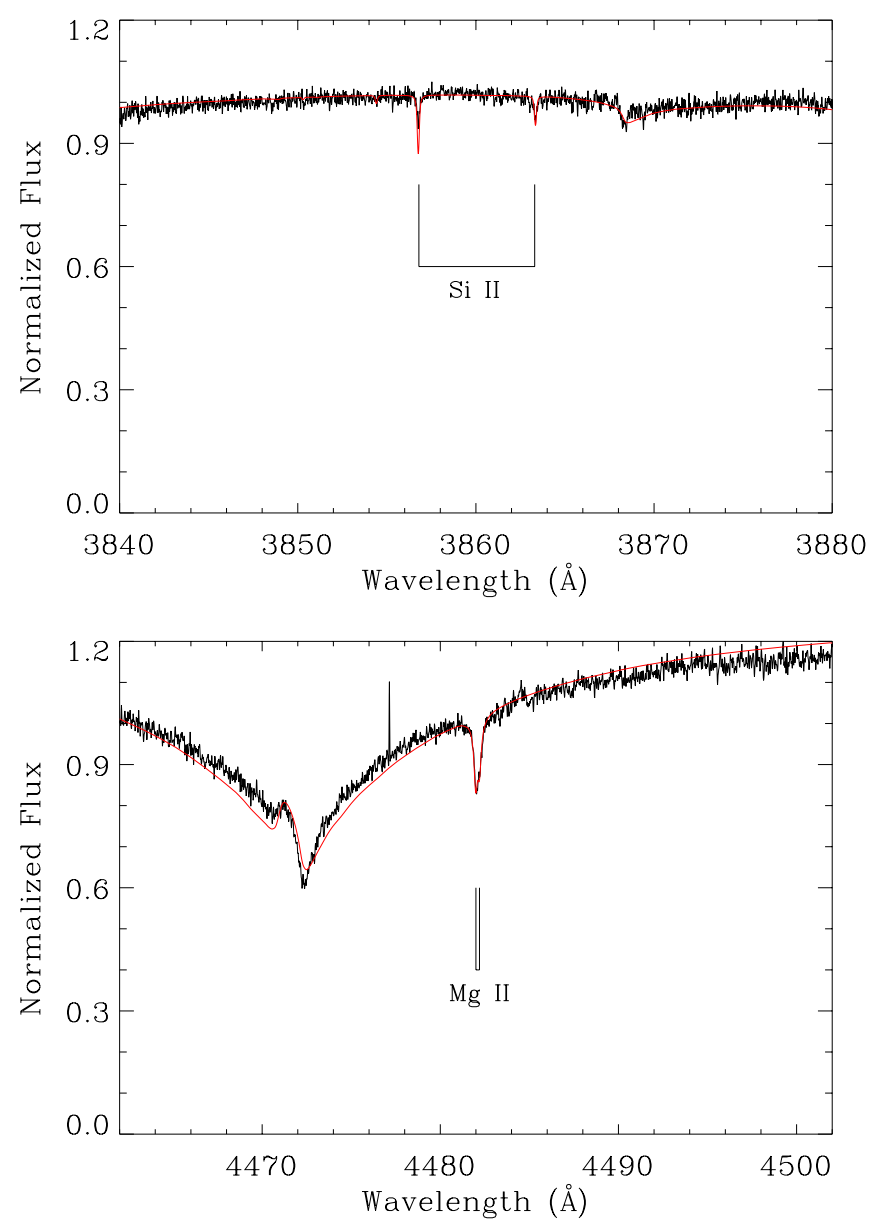

2007) and consistent with closely orbiting debris resulting from the tidal destruction of a planetary body such as a large asteroid.

\subsection{Atmospheric Heavy Elements}

GD 61 was observed using the High Resolution Echelle Spectrograph (HIRES; Vogt et al. 1994) at the Keck I telescope on Mauna Kea. Spectra covering 3130-5960 A (with gaps at 4004-4064 and 4992-5071 $\AA$ ) were acquired on 2008 February 13, and the $R \sim 40,000$ data were reduced and analyzed following Klein et al. (2010). Heavy elements detected in the HIRES spectrum are $\mathrm{Mg}_{\mathrm{I}}(3838 \AA), \mathrm{Mg}_{\text {II }}(4481 \AA)$, Si II $(3586,3863,4128$, and $4131 \AA)$, and Ca II $(3159,3179,3934$, and $3969 \AA$ ). Figure 2 displays a few important sections of the spectrum.

Element abundances were derived from comparisons of equivalent widths in the data with those of stellar atmosphere models, with upper limits established from the absence of model-predicted features. The average $\mathrm{Ca}$ abundance was weighted by feature strength, giving most weight to the wellstudied $\mathrm{Ca}$ II $\mathrm{H} \& \mathrm{~K}$ resonance lines. For $\mathrm{Mg}$ and $\mathrm{Si}$, the abundances for the sets of lines were combined in a direct average. The uncertainties have been calculated in a manner similar to that described in Klein et al. (2010), by varying the model temperature and surface gravity by $\pm 400 \mathrm{~K}$ and $\pm 0.3 \mathrm{dex}$, respectively.
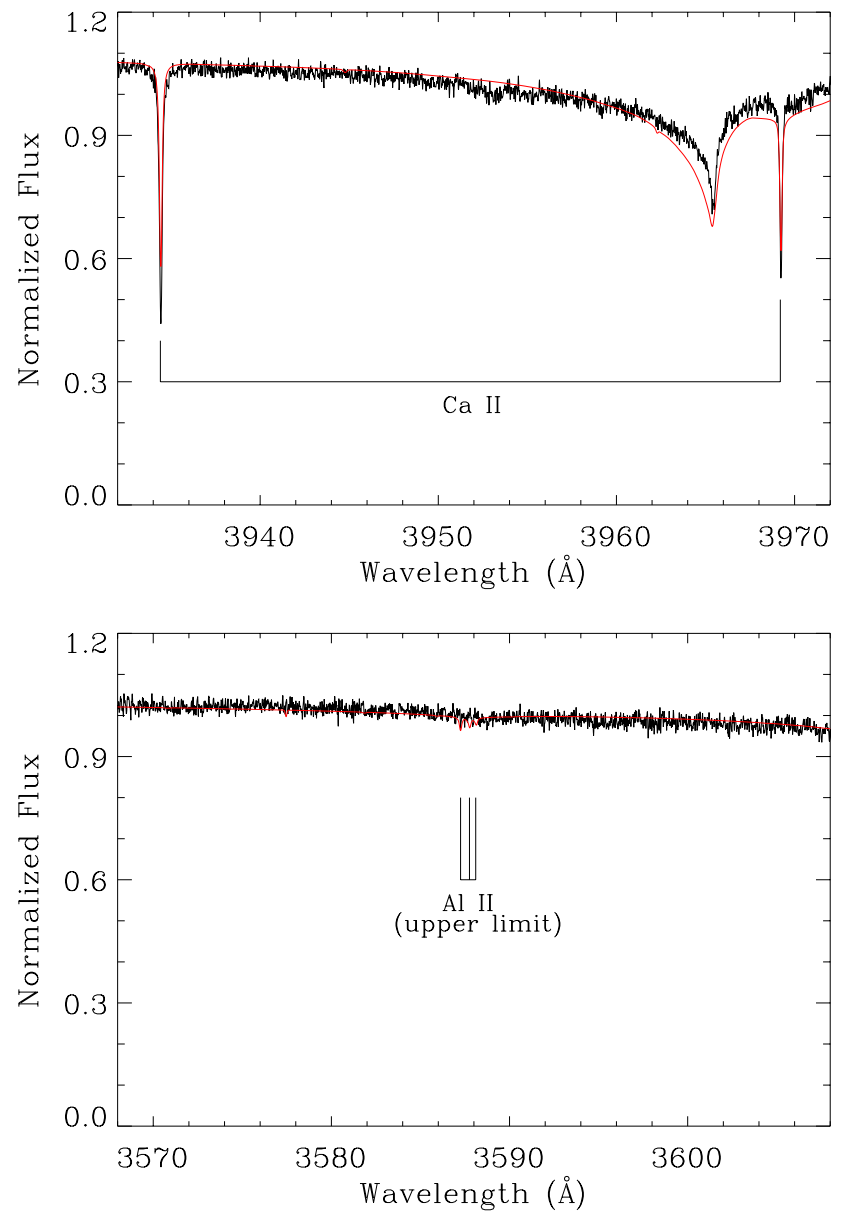

Figure 2. HIRES data for GD 61, showing regions containing key element detections and upper limits. The unsmoothed data are shown in black and the model overplotted in red. There are unavoidable and slight imperfections in the flux calibration, and these can be seen relative to the model; however, these do not affect the abundance determinations.

(A color version of this figure is available in the online journal.) 
Table 1

Observed and Modeled Properties of GD 61

\begin{tabular}{rlccc}
\hline \hline \multicolumn{2}{c}{ Parameter } & \multicolumn{3}{c}{ Fluxes } \\
\cline { 3 - 5 } & & Filter & $\lambda_{\text {eff }}(\mu \mathrm{m})$ & $F_{v}(\mathrm{mJy})$ \\
\hline$T_{\text {eff }}$ & $17,280 \mathrm{~K}$ & $U$ & 0.37 & $5.87 \pm 0.27$ \\
$\log g\left(\mathrm{~cm} \mathrm{~s}^{-2}\right)$ & 8.20 & $B$ & 0.44 & $5.36 \pm 0.27$ \\
$d$ & $50.1 \mathrm{pc}$ & $V$ & 0.55 & $4.41 \pm 0.22$ \\
$M$ & $0.71 M_{\odot}$ & $r^{\prime}$ & 0.64 & $3.84 \pm 0.28$ \\
$M_{\mathrm{ms}}$ & $3.0 M_{\odot}(\mathrm{A} 0 \mathrm{~V})$ & $J$ & 1.24 & $1.42 \pm 0.07$ \\
Total age & $0.6 \mathrm{Gyr}$ & $H$ & 1.66 & $0.81 \pm 0.07$ \\
$\left(\mu_{\alpha}, \mu_{\delta}\right)$ & $(-19,-102) \mathrm{mas} \mathrm{yr}^{-1}$ & $K_{s}$ & 2.16 & $0.72 \pm 0.09$ \\
$z_{\mathrm{g}} c$ & $+41 \mathrm{~km} \mathrm{~s}^{-1}$ & IRAC 2 & 4.49 & $0.267 \pm 0.013$ \\
$v_{\mathrm{rad}}$ & $+17 \mathrm{~km} \mathrm{~s}^{-1}$ & IRAC 4 & 7.87 & $0.149 \pm 0.009$ \\
$(U, V, W)$ & $(10,-4,-13) \mathrm{km} \mathrm{s}^{-1}$ & IRS Blue & 15.8 & $0.098 \pm 0.010$
\end{tabular}

Notes. Temperature and surface gravity are from Desharnais et al. (2008) and provide a good fit to the HIRES spectrum. The total age estimate is the sum of the white dwarf cooling age (Fontaine et al. 2001) and main-sequence lifetime (Hurley et al. 2000). The radial velocity is the difference between the average measured line velocity of $+58 \mathrm{~km} \mathrm{~s}^{-1}$ and the gravitational redshift of the star. Short-wavelength photometry and proper motion are taken from the literature and available catalogs.

Prior observations of GD 61 with the Far-Ultraviolet Spectroscopic Explorer (FUSE) were obtained and analyzed in detail by Desharnais et al. (2008). In order of decreasing abundance, these data reveal trace amounts of $\mathrm{H}, \mathrm{O}, \mathrm{Si}$, and $\mathrm{Fe}$ in the He-dominated atmosphere of the white dwarf. Modeled stellar parameters found by Desharnais et al. (2008) are listed in Table 1 and were found to fit the He lines in the HIRES spectrum of GD 61 quite well overall. Furthermore, these parameters are consistent with the ultraviolet through infrared photometric energy distribution of the star shown in Figure 1, and were adopted for all purposes in this work, including the determination of metal abundances and upper limits from the HIRES data set.

Only Si is detected in both the FUSE and HIRES data sets, and the independent analyses agree rather well, as shown in Table 2. Also, the upper limit $\mathrm{Fe}$ abundance determined from the HIRES spectrum is consistent with its reported FUSE detection. In order to preserve the metal-to-metal ratios found for each spectrum independently, all heavy element ratios were tied to the weighted average of the FUSE and HIRES Si abundances. Table 2 lists the HIRES, FUSE, and adopted heavy element abundances relative to $\mathrm{He}$, while carrying the errors distinct to each data set.

\subsection{Infalling Debris from a Destroyed Minor Planet}

Rather notably, $\mathrm{O}$ is the most abundant heavy element in GD 61, whereas only a strict upper limit for $\mathrm{C}$ could be determined from the ultraviolet data. Lacking information on its circumstellar environment, Desharnais et al. (2008) had to invoke convective dredge-up combined with a highly unusual chemical profile of the white dwarf core to account for the extreme O/C > 1000 found in GD 61. However, all white dwarfs suspected of harboring dredged-up core material exhibit atmospheric $\mathrm{C}$ and effective temperatures near or below $12,000 \mathrm{~K}$ (Dufour et al. 2005), including the recently detected class of O-rich white dwarfs (Gänsicke et al. 2010). At 17,300 K, the depth of the convective envelope in GD 61 is only $2.5 \%$ of the maximum depth attained in He atmosphere white dwarfs (Koester 2009), which occurs between 12,000 and 10,000 K (Pelletier et al. 1986). Furthermore, dredge-up cannot account for detectable amounts of $\mathrm{Si}, \mathrm{Ca}$, and $\mathrm{Fe}$ in the atmosphere of a white dwarf, and Desharnais et al. (2008) concluded that GD 61 must have accreted these metals from the interstellar medium. However, they did not consider that metals are locked up in dust grains within the interstellar medium, and this dust is composed of O-rich silicates (Draine 2003). Such material provides a natural source for the observed $\mathrm{O}$ if interstellar silicates were accreted.

The warm dust orbiting metal-rich white dwarfs and mature stars in general is rich in silicate minerals (Jura et al. 2009a; Lisse et al. 2008), and hence a large deposition of $\mathrm{Si}$ and $\mathrm{O}$ should result from the accretion of this debris, in excellent qualitative agreement with the polluted atmosphere of GD 61. Accretion from the closely orbiting dust disk must be ongoing due to viscous dissipation, primarily gas drag at the inner disk edge where dust grains rapidly sublimate (Jura 2008). Material found orbiting within 20 stellar radii demonstrates that the white dwarf is currently accreting from this reservoir and provides a natural explanation for all the photospheric heavy elements, including $\mathrm{O}$.

\section{RESULTS}

\subsection{Chemical Abundances of the Debris}

The heavy elements observed in GD 61 represent the chemical constituency of its circumstellar disk, and their abundance ratios provide constraints on the nature of the minor planet pulverized into the orbiting debris. These elements reside in the $2.15 \times$

Table 2

Heavy Elements in the Disrupted Minor Planet Orbiting GD 61

\begin{tabular}{lcccccc}
\hline \hline & \multicolumn{3}{c}{$\log [n(\mathrm{Z}) / n(\mathrm{He})]$} & \multicolumn{2}{c}{ Early Phase } & Steady State \\
\cline { 3 - 5 } Element & $t_{\text {diff }}\left(10^{5} \mathrm{yr}\right)$ & HIRES & $F U S E$ & Adopted & $M_{\mathrm{z}}\left(10^{21} \mathrm{~g}\right)$ & $M_{\mathrm{z}}\left(10^{21} \mathrm{~g}\right)$ \\
\hline $\mathrm{H}$ & $\infty$ & $-3.98 \pm 0.02$ & $\ldots$ & $-3.98 \pm 0.02$ & 5.615 & 5.615 \\
$\mathrm{C}$ & 1.245 & $<-8.8$ & $\ldots$ & $<-8.93$ & $<0.001$ & $\ldots$ \\
$\mathrm{O}$ & 0.938 & $\ldots$ & $-5.8 \pm 0.2$ & $-5.93 \pm 0.20$ & 1.017 & 0.873 \\
$\mathrm{Mg}$ & 0.794 & $-6.65 \pm 0.18$ & $\ldots$ & $-6.63 \pm 0.18$ & 0.308 & 0.312 \\
$\mathrm{Al}$ & 0.685 & $<-7.2$ & $\ldots$ & $<-7.18$ & $<0.097$ & $\ldots$ \\
$\mathrm{Si}$ & 0.650 & $-6.85 \pm 0.09$ & $-6.7 \pm 0.2$ & $-6.83 \pm 0.08$ & 0.225 & 0.278 \\
$\mathrm{Ca}$ & 0.501 & $-7.90 \pm 0.19$ & $\ldots$ & $-7.88 \pm 0.19$ & 0.029 & 0.046 \\
$\mathrm{Ti}$ & 0.436 & $<-9.1$ & $\ldots$ & $<-9.08$ & $<0.002$ & $\ldots$ \\
$\mathrm{Cr}$ & 0.396 & $<-9.0$ & $\ldots$ & $<-8.98$ & $<0.003$ & $\ldots$ \\
$\mathrm{Fe}$ & 0.361 & $<-7.5$ & $-7.6 \pm 0.2$ & $-7.73 \pm 0.20$ & 0.056 & 0.125 \\
$\mathrm{Ni}$ & 0.346 & $<-7.6$ & $\cdots$ & $<-7.58$ & $<0.084$ & $\cdots$ \\
\hline
\end{tabular}

Notes. Adopted abundances, upper limits, and errors are discussed in Section 2. The last two columns list the cumulative mass of each element residing in the convection zone of GD 61 for the early phase and steady state cases. Thus, the accreted metals in GD 61 total a minimum of $1.64 \times 10^{21} \mathrm{~g}$, while an ongoing event older than $2.5 \times 10^{5} \mathrm{yr}$ implies a total accreted mass exceeding $10^{23} \mathrm{~g}$. 
Table 3

Assessment of Oxygen Mass Fractions in GD 61

\begin{tabular}{lccccc}
\hline \hline \multirow{2}{*}{ Oxygen Carrier } & \multicolumn{2}{c}{ Early Phase } & & \multicolumn{2}{c}{ Steady State } \\
\cline { 2 - 3 } & Fraction & Error $^{\mathrm{a}}$ & & Fraction & Error $^{\mathrm{a}}$ \\
\hline $\mathrm{SiO}_{2}$ & 0.252 & 0.110 & & 0.363 & 0.158 \\
$\mathrm{MgO}$ & 0.200 & 0.115 & & 0.236 & 0.136 \\
$\mathrm{Al}_{2} \mathrm{O}_{3}$ & $<0.084$ & $\cdots$ & & $<0.115$ & $\ldots$ \\
$\mathrm{FeO}^{\mathrm{b}}$ & 0.016 & 0.010 & & 0.041 & 0.025 \\
$\mathrm{CaO}$ & 0.011 & 0.007 & & 0.021 & 0.013 \\
\hline Excess (Chondritic Al) & 0.498 & 0.223 & & 0.307 & 0.304 \\
Excess (Maximum Al) & 0.437 & 0.246 & & 0.224 & 0.334 \\
\hline
\end{tabular}

Notes.

a Standard deviations resulting from all possible permutations of individual element abundances and associated errors.

$\mathrm{b}$ The mass fraction of Fe is insufficient for the outcome to be affected by significant amounts of $\mathrm{Fe}_{2} \mathrm{O}_{3}$, which is only present in significant quantities in some Earth basalts, but not in the bulk of meteorites, including Lunar meteorites and Eucrites (Basaltic Volcanism Study Project 1981).

$10^{26} \mathrm{~g}$ stellar convection zone, where they are thoroughly mixed and have sinking timescales on the order of $10^{4.5} \mathrm{yr}$ (Koester 2009). Table 2 lists the current (early phase) abundances and masses of each heavy element detected in the star, as well as upper limits established from the HIRES and FUSE spectra. Based on these values, and because metals continually sink below the photosphere, the destroyed planetary body had a minimum mass of $1.64 \times 10^{21} \mathrm{~g}$, roughly equivalent to an asteroid $110 \mathrm{~km}$ in diameter.

If the disk age is less than around $10^{3} \mathrm{yr}\left(\ll t_{\text {diff }}\right)$, then insufficient time has passed for any metal to substantially diffuse below the photosphere. Observable signatures of the element ratios and the total accreted mass of the disrupted asteroid are preserved in the star during this early phase of accretion. On the other hand, if the disk age approaches or exceeds several $t_{\text {diff }}$, a steady state balance between accretion and diffusion is achieved for each heavy element (Koester \& Wilken 2006). In either case, the abundance ratios and minimum element masses in the minor planet can be derived analytically, without knowing the exact age of the disk, and are listed in Table 2.

\subsubsection{Potential Evidence of $\mathrm{H}_{2} \mathrm{O}$}

Both the early phase and steady state scenarios predict that the total mass of observed heavy elements in GD 61 is dominated by $\mathrm{O}$. If all the $\mathrm{Mg}, \mathrm{Si}, \mathrm{Ca}$, and $\mathrm{Fe}$ were originally contained in $\mathrm{MgO}, \mathrm{SiO}_{2}, \mathrm{CaO}$, and $\mathrm{FeO}$, as in chondrites and the rocky material of the inner solar system, the nominal FUSE and HIRES abundances indicate a significant $\mathrm{O}$ excess. Table 3 explores the fraction of $\mathrm{O}$ contained in these metal oxides, together with upper limit and chondritic $\mathrm{Al}$ delivered as $\mathrm{Al}_{2} \mathrm{O}_{3}$. Even in the case where $\mathrm{Al}$ is present at its upper limit abundance as determined by HIRES, there is a nominal excess mass of $\mathrm{O}$ by $22 \%$ or $44 \%$ in the steady state or early phases, respectively. However, at the minimum values of $\mathrm{O} / \mathrm{Mg}$ and $\mathrm{O} / \mathrm{Si}$ permitted by the errors, the $\mathrm{O}$ can be accounted for completely by dry mineral oxides, and thus an excess cannot be confirmed with confidence using the present data.

The potential for excess $\mathrm{O}$ in GD 61 is of interest because the most natural explanation would be that extra $\mathrm{O}$ was originally bound in water ice representing a significant fraction of the total mass of the minor planet, $25 \%-35 \%$ in the steady state. It is possible for water to survive the post-main-sequence evolution of the star, if sufficiently buried within its parent body (Jura \& $\mathrm{Xu}$ 2010). Upon shattering during a close approach with a white dwarf, any water ice (and volatiles) would rapidly sublimate but eventually fall onto the star-the feeble luminosity of white dwarfs is insufficient to remove even light gases by radiation pressure (Farihi et al. 2008).

If the system is still in an early phase of accretion, the potential for water is enhanced, as the implied $\mathrm{O}$ abundance of the accreted material is then at its highest. Given that disks at white dwarfs appear to be long-lived-and in this way potentially analogous to planetary rings in the solar system-with lifetimes possibly exceeding $10^{6}$ yr (Klein et al. 2010; Jura et al. 2009b; Jura 2008; Farihi et al. 2008), the chance of catching the star in the early phase is small. Regardless, the primary source of uncertainty for a water-rich accretion event at GD 61 is the abundance errors on $\mathrm{O}, \mathrm{Mg}$, and $\mathrm{Si}$.

\subsubsection{Possible Evidence of Differentiation}

GD 61 is polluted by material that is deficient in Fe relative to both $\mathrm{Mg}$ and $\mathrm{Si}$. Chondritic and solar ratios among these elements are near unity: $\mathrm{Fe} / \mathrm{Mg}=0.83, \mathrm{Fe} / \mathrm{Si}=0.85$ (Lodders 2003). These same ratios in GD 61 are $0.08,0.13$ in the early phase and $0.18,0.23$ in the steady state, respectively. In either case, the accreted material was depleted in Fe relative to the material of the inner solar system.

If the remnant planetary system at GD 61 formed at roughly chondritic element ratios, then some process must have depleted Fe from the parent body that now exists as a circumstellar ring of debris. Exactly such an occurrence is the favored hypothesis for the origin of the Moon. Near the end of terrestrial planet formation, a Mars-sized body impacted the Earth, ejecting the mantle of the impactor while the cores of the two bodies liquified and fused (Canup \& Asphaug 2001). If the event happened sufficiently late that both bodies were largely differentiated, the ejected mantle would be relatively Fe-poor, giving rise to the observed deficiency in the Moon.

It is unknown if extrasolar terrestrial bodies form at chondritic compositions or if significant variations exist. Studying destroyed rocky planetary bodies at white dwarfs will help shed light on this important and interesting question. It is conceivable that the minor (or major) planet that gave rise to the metals in GD 61 was formed with a distinctly non-solar Fe abundance.

\section{OUTLOOK}

The present uncertainties in the $\mathrm{O} / \mathrm{Mg}$ and $\mathrm{O} / \mathrm{Si}$ ratios in GD 61 permit an interpretation where the accretion of water is not required to account for its O-rich atmosphere. In order to reduce these errors, a spectrum containing all the Table 2 detected elements is needed, whereby metal abundances can be tied to $\mathrm{O}$ (rather than $\mathrm{He}$ ) and a confident assessment of its oxide contribution can be made.

If the excess $\mathrm{O}$ can be confirmed with better data, the accretion of a water-rich asteroid is the most natural explanation for the circumstellar debris detected by Spitzer and the pattern of elements in the disk-polluted star. If all the trace H in GD 61 was delivered by a single asteroid that has polluted the star for many diffusion timescales (metals continuously sink, while $\mathrm{H}$ floats), then the parent body contained $5.0 \times 10^{22} \mathrm{~g}$ of water. If such a minor planet were $25 \%-35 \%$ water by mass as inferred from the steady state analysis and nominal $\mathrm{O}$ abundance, then its total mass would be $(1.4-2.0) \times 10^{23} \mathrm{~g}$ and approaching that of Vesta, the second most massive asteroid in the solar 
system. Such a large mass of orbiting material is permitted by the optically thick disk model shown in Figure 1 (Jura 2003), while the steady state scenario implies that a comparable mass has already been accreted by the star.

GD 61 appears to have a relatively young total age. This picture is supported by the spectroscopically determined mass and also by its three-dimensional space velocity vector $(U, V, W)$ in Table 1 . Its current mass of $0.71 M_{\odot}$ implies a likely descent from a main-sequence A0 V star of around $3.0 M_{\odot}$ (Williams et al. 2009; Kalirai et al. 2008; Dobbie et al. 2006), and its overall mild velocity is consistent with relatively young, thin disk kinematics. A total age of $0.6 \mathrm{Gyr}$ is inferred from its mainsequence lifetime plus cooling age (Fontaine et al. 2001; Hurley et al. 2000). Thus, the debris orbiting GD 61 is material associated with a rocky planetary system formed about a relatively young, intermediate-mass star.

Recently, significant amounts of water have potentially been identified in the asteroid belt of the solar system, via main belt comet activity (Hsieh \& Jewitt 2006) and surface water on Themis (Campins et al. 2010; Rivkin et al. 2010). These discoveries have important implications for the delivery of water to a dry Earth, a task that appears most readily accomplished by primordial asteroids-planetary embryos analogous to Ceres - in the outer main belt (Morbidelli et al. 2000). Ceres itself is thought to harbor significant water ice in its interior; its density and dynamical modeling suggest a fraction of $25 \%$ water by mass (Thomas et al. 2005). The white dwarf GD 61 is surrounded by a ring of dust, and the totality of its atmospheric pollution can be understood as the tidal destruction of a parent body similar in mass to Vesta. If confirmed, the potential $\mathrm{O}$ excess would suggest a water content analogous to Ceres. These two largest solar system asteroids are (just) massive enough to be differentiated, and the metals in GD 61 suggest that its circumstellar debris may have originated in a similarly massive, rocky exoplanetary body.

The authors thank the anonymous referee for a careful reading and a report that improved the manuscript. This work is based in part on observations made with the Spitzer Space Telescope, which is operated by the Jet Propulsion Laboratory, California Institute of Technology under a contract with NASA. Some of the data presented herein were obtained at the W. M. Keck Observatory, which is operated as a scientific partnership among the California Institute of Technology, the University of California, and NASA. This publication makes use of data products from the Two Micron All Sky Survey.

Facility: Spitzer (IRAC); Keck:I (HIRES)

\section{REFERENCES}

Basaltic Volcanism Study Project 1981, Basaltic Volcanism on the Terrestrial Planets (New York: Pergamon)
Brinkworth, C. S., Gänsicke, B. T., Marsh, T. R., Hoard, D. W., \& Tappert, C. 2009, ApJ, 696, 1402

Campins, H., et al. 2010, Nature, 464, 1320

Canup, R. M., \& Asphaug, E. 2001, Nature, 412, 708

Debes, J. H., \& Sigurdsson, S. 2002, ApJ, 572, 556

Desharnais, S., Wesemael, F., Chayer, P., Kruk, J. W., \& Saffer, R. A. 2008, ApJ, 672,540

Dobbie, P. D., et al. 2006, MNRAS, 369, 383

Draine, B. T. 2003, ARA\&A, 41, 241

Dufour, P., Bergeron, P., \& Fontaine, G. 2005, ApJ, 627, 404

Farihi, J., Barstow, M. A., Redfield, S., Dufour, P., \& Hambly, N. C. 2010a, MNRAS, 404, 2123

Farihi, J., Jura, M., Lee, J. E., \& Zuckerman, B. 2010b, ApJ, 714, 1386

Farihi, J., Jura, M., \& Zuckerman, B. 2009, ApJ, 694, 805

Farihi, J., Zuckerman, B., \& Becklin, E. E. 2005, AJ, 130, 2237

Farihi, J., Zuckerman, B., \& Becklin, E. E. 2008, ApJ, 674, 431

Fazio, G. G., et al. 2004, ApJS, 154, 10

Fontaine, G., Brassard, P., \& Bergeron, P. 2001, PASP, 113, 409

Gänsicke, B. T., Koester, D., Girven, J., Marsh, T. R., \& Steeghs, D. 2010, Science, 327, 188

Gänsicke, B. T., Koester, D., Marsh, T. R., Rebassa-Mansergas, A., \& Southworth, J. 2008, MNRAS, 391, L103

Gänsicke, B. T., Marsh, T. R., Southworth, J., \& Rebassa-Mansergas, A. 2006, Science, 314, 1908

Houck, J. R., et al. 2004, ApJS, 154, 18

Hsieh, H. H., \& Jewitt, D. 2006, Science, 312, 561

Hurley, J. R., Pols, O. R., \& Tout, C. A. 2000, MNRAS, 315, 543

Jura, M. 2003, ApJ, 584, L91

Jura, M. 2008, AJ, 135, 1785

Jura, M., Farihi, J., \& Zuckerman, B. 2007, ApJ, 663, 1285

Jura, M., Farihi, J., \& Zuckerman, B. 2009a, AJ, 137, 3191

Jura, M., Muno, M., Farihi, J., \& Zuckerman, B. 2009b, ApJ, 699, 1473

Jura, M., \& Xu, S. 2010, AJ, 140, 1129

Kalirai, J. S., Hansen, B. M. S., Kelson, D. D., Reitzel, D. B., Rich, R. M., \& Richer, H. B. 2008, ApJ, 676, 594

Klein, B., Jura, M., Koester, D., Zuckerman, B., \& Melis, C. 2010, ApJ, 709, 950

Koester, D. 2009, A\&A, 498, 517

Koester, D., Napiwotzki, R., Voss, B., Homeier, D., \& Reimers, D. 2005, A\&A, 439, 317

Koester, D., \& Wilken, D. 2006, A\&A, 453, 1051

Lisse, C. M., Chen, C. H., Wyatt, M. C., \& Morlok, A. 2008, ApJ, 673, 1106

Lodders, K. 2003, ApJ, 591, 1220

Morbidelli, A., Chambers, J., Lunine, J. I., Petit, J. M., Robert, F., Valsecchi, G. B., \& Cyr, K. E. 2000, Meteorit. Planet. Sci., 35, 1309

Patten, B. M., et al. 2006, ApJ, 651, 502

Pelletier, C., Fontaine, G., Wesemael, F., Michaud, G., \& Wegner, G. 1986, ApJ, 307,242

Reach, W. T., Kuchner, M. J., von Hippel, T., Burrows, A., Mullally, F., Kilic, M., \& Winget, D. E. 2005, ApJ, 635, L161

Reach, W. T., Lisse, C., von Hippel, T., \& Mullally, F. 2009, ApJ, 693, 697

Rivkin, A. S., \& Emery, J. P. 2010, Nature, 464, 1322

Sion, E. M., Aannestad, P. A., \& Kenyon, S. J. 1988, ApJ, 330, L55

Thomas, P. C., Parker, J. W., McFadden, L. A., Russell, C. T., Stern, S. A., Sykes, M. V., \& Young, E. F. 2005, Nature, 437, 224

Vogt, S. S., et al. 1994, Proc. SPIE, 2198, 362

von Hippel, T., Kuchner, M. J., Kilic, M., Mullally, F., \& Reach, W. T. 2007, ApJ, 662,544

Werner, M. W., et al. 2004, ApJS, 154, 1

Williams, K. A., Bolte, M., \& Koester, D. 2009, ApJ, 693, 355

Zuckerman, B., Koester, D., Melis, C., Hansen, B. M. S., \& Jura, M. 2007, ApJ, 671,872

Zuckerman, B., Koester, D., Reid, I. N., \& Hünsch, M. 2003, ApJ, 596, 477 